\title{
Monitoring reliability of sensors in an array by neural networks
}

\author{
M. Pardo ${ }^{a}$ G. Faglia ${ }^{a}$ G. Sberveglieri ${ }^{a}$ M. Corte $^{b}$ F. Masulli ${ }^{c}$ \\ M. Riani ${ }^{\mathrm{b}}$ \\ ${ }^{\mathrm{a}}$ INFM, Dept.Chemistry and Physic for Materials - University of Brescia - \\ Via Valotti 9 - 25133 Brescia -Italy. \\ ${ }^{\mathrm{b}}$ INFM, DIFI Dept. of Physics - University of Genova \\ Via Dodecaneso 33, 16146 Genova -Italy. \\ ${ }^{\mathrm{c}}$ INFM, DISI Dept. of Computer and Information Sciences - University of Genova \\ Via Dodecaneso 35, 16146 Genova -Italy.
}

\begin{abstract}
The correlation between the responses of five semiconductor thin films sensors to $\mathrm{CO}-\mathrm{NO}_{2}$ mixtures is exploited to detect a possible malfunctioning of one of the sensors during operation. To this end, at every time instant, the current flowing in each single sensor is estimated as a function of the current flowing in the remaining ones. The estimation is accomplished using the regression ability of five artificial neural networks (ANN), one for each sensor. We show, in the case of a simulated transient malfunctioning, how it is possible to detect on-line which is the sensor that is not working properly. Further, after a fault has been detected, the estimation replaces the damaged sensor response. In this way the concentration prediction performed by other ANNs which need the responses of all the sensors- can proceed until the damaged sensor has been replaced.
\end{abstract}

Key words: Fault detection; Error compensation; Neural networks; Thin films.

\section{Introduction}

Sensor arrays together with pattern recognition techniques have been shown to distinguish between different gases, both individually $[1,2,3,4]$ and in a mixture $[5,6,7,8]$. Moreover, even in the case of mixtures it has been possible to predict the components' concentrations. A chief application of this capability is to environmental monitoring.

Preprint submitted to Elsevier Preprint 9th February 2000 
In the cited papers it is usually assumed that sensors operate correctly. For on-line application of sensor arrays in real world problems, however, it is of great interest to be able to check whether sensors do function correctly (fault detection) and, in case of malfunctioning, to compensate this malfunctioning until the damaged sensor is substituted.

In $[9,10]$, we compared two neural network based approaches to the estimation of $\mathrm{NO}_{2}$ and $\mathrm{CO}$ concentrations in mixtures with humid air. By studying the prediction ability of a five sensors array, we found out that the estimation error on the test set increased only slightly using four sensors (while the increase with three sensors was sensible). This is due to the correlation between the sensors (see sect. 3.1).

In the present contribution we show how the correlation between different sensor can be used for fault detection and compensation. In this way, in spite of trying to minimize the number of sensors for prediction purposes by eliminating e.g. sensors which have a low (or equal) loading on the first principal components, we exploit the redundancy present in the different responses. The technique we prose exploits the concept of a virtual sensor, see e.g. [11], with the aim of increasing the system reliability. To our knowledge no similar approach has been proposed in the chemical sensor field.

The structure of the paper is as follows. In the next Sect. we give details on the sensors array and on its characterization in the quantitative measurement of component concentration in a gas mixture. In Sect. 3.1 we analyze the correlation among sensors, and we show the characteristics of neural crossestimators of their conductances. The system for reliability control of a sensor array based on these cross-estimators is presented in Sect. 3.2. Experimental results on our sensor array are presented and discussed in Sect. 4. Conclusions are drawn in Sect. 5 .

\section{Experimental}

An array of five tin oxide thin films have been deposited through the R.G.T.O. technique over alumina substrates [12]. A Pt thin film was deposited on the back of the substrate as a heater and temperature sensor. In order to enhance the array selectivity, ultra thin films of metal catalysts ( $\mathrm{Au}$ and $\mathrm{Pt}$ ) were deposited over the sensor surface. The change of conductance has been monitored by a volt-amperometric technique at constant bias [13]. The array conductance response to nitrogen dioxide (0.2 - $0.6-2-4 \mathrm{ppm})$ and carbon monoxide (0 -25 -50 -100 -200 ppm) mixtures was examined. These values are close to the alarm levels for environmental protection in many European countries. During the characterization relative humidity was set to $r h=30 \%$ 
at $T=20^{\circ} \mathrm{C}$. Two runs of measurements -each constituted by 20 different concentration mixtures- were completed in order to verify the reproducibility of the array performance. Moreover, since tin oxide sensitivity to $\mathrm{NO}_{2}$ is enhanced at lower temperature while the sensitivity to $C O$ is enhanced at higher temperature, the local operating temperature of two sensors was kept equal to $600 \mathrm{~K}(\mathrm{Pt}, \mathrm{Au})$, while the others were operated at $700 \mathrm{~K}$ (bare, Pt, Au).

\section{Method}

\subsection{Estimation of sensors output}

We would like to correlate the sensor responses by estimating the functional dependences (for every time instant):

$$
\begin{aligned}
& i_{A}=f_{A}\left(i_{D}, i_{E}, i_{G}, i_{H}\right) \\
& i_{D}=f_{D}\left(i_{A}, i_{E}, i_{G}, i_{H}\right) \\
& i_{E}=f_{E}\left(i_{A}, i_{D}, i_{G}, i_{H}\right) \\
& i_{G}=f_{G}\left(i_{A}, i_{D}, i_{E}, i_{H}\right) \\
& i_{H}=f_{H}\left(i_{A}, i_{D}, i_{E}, i_{G}\right)
\end{aligned}
$$

In order to evaluate the correlation among the sensors' conductances, we began by assuming a linear dependence. A multiple linear regression analysis shows that the multiple linear correlation coefficients are all greater than 0.89 . We subsequently tried to estimate the real nonlinear functional dependence with neural networks. In this application we use the ability of MLPs to behave as universal function approximators [14].

Every neural networks has four inputs, eight hidden neurons and one output neuron; the units of the hidden and output layers have a sigmoid-shaped activation function, while the input units do not perform any calculation. The training was performed by pattern using the standard backpropagation algorithm with learning rate $\eta=0.1$ and a momentum term $\alpha=0.9$. To avoid misleading effects due to the particular sequence of the measurements, the presentation order of the patterns was randomized. The networks were trained using the first sequence of measurements as training set and the second one, that was obtained 2 days after the first, as test set. Although the gas mixtures are the same as in the first measurement series, the sensors' responses after 2 days present a temporal drift because the sensors had not been aged previously. 
As shown in Tab. 1, the obtained current estimation is good for every sensor. In Fig. 1 we show in graphical form the high quality result obtained on the test set for sensor A. The predicted and the measured curves are very often superimposed on each other. Similarly, in Fig. 2 the result for sensor H is shown as the scatter plot of the pairs (predicted current, measured current): this shows that the approximation is very good, because all the points lay very near to the ideal case represented by the function $y=x$, with a small variance and no noticeable bias.

\subsection{Modular MLP system}

The result in the preceding section suggests a viable approach to monitoring the working state and to detecting possible malfunctioning of sensors. This can be done by using five MLP networks, each of them estimating the current flowing in one sensor as non-linear function of the currents flowing in the other ones at the same time. As sketched in Fig. 3, each estimator has 4 inputs and one output; e.g., Estim A has D, E, G, H as inputs and estimates the current flowing in A.

In order to control the reliability of the array of sensors we can continuously compare the current flowing in each sensor (Fig. 3 left) and its estimate provided by the neural networks array (Fig. 3 right) with appropriate testing units (Fig. 3 bottom). In these units we can implement various criteria in order to detect different kind of faults. For example, by simply considering the difference between predicted output and actual output (estimation error) as a signature of the correct working state of sensors, we can detect transient faults. Further, long time drifts can be detected checking if the estimation errors are Normal distributed.

\section{Simulation and discussion}

We show now the effect of a simulated transient malfunctioning in sensor $\mathrm{H}$ obtained by changing the measured current at three time points (see Fig. 4).

As a first step of the fault detection algorithm, using the already trained 
MLPs, we compute

$$
\begin{aligned}
& \hat{i}_{A}=\hat{f}_{A}\left(i_{D}, i_{E}, i_{G}, i_{H}\right) \\
& \hat{i}_{D}=\hat{f}_{D}\left(i_{A}, i_{E}, i_{G}, i_{H}\right) \\
& \hat{i}_{E}=\hat{f}_{E}\left(i_{A}, i_{D}, i_{G}, i_{H}\right) \\
& \hat{i}_{G}=\hat{f}_{G}\left(i_{A}, i_{D}, i_{E}, i_{H}\right) \\
& \hat{i}_{H}=\hat{f}_{H}\left(i_{A}, i_{D}, i_{E}, i_{G}\right)
\end{aligned}
$$

where $i_{A}, i_{D}, i_{G}, i_{E}$ and $i_{H}$ are the measured values of the currents and $\hat{i}_{A}$, $\hat{i}_{D}, \hat{i}_{G}, \hat{i}_{E}$ and $\hat{i}_{H}$ the estimated ones.

Comparing $i_{A}$ with $\hat{i}_{A}, i_{D}$ with $\hat{i}_{D}$ and so on, we find unusual differences between the estimated and measured values at three points (Fig.5). This behavior has two different sources:

- in the cases A, D, E and G, the estimated output is incorrect because the input corresponding to sensor $\mathrm{H}$ is wrong

- on the other side, Estim $H$ gives a correct value, that is a value that $H$ would have if it worked correctly. The estimation error is unusual high due to the transient fault in $i_{H}$.

A priori all we can conclude is that (at least) one of the sensors is not working as expected, but we cannot yet say which one it is. To find out which is the malfunctioning sensor we adopt a simple strategy. We substitute one of the sensors in turn with its neural model: beginning with sensor A we compute $\hat{i}_{A}=f_{A}\left(i_{D}, i_{E}, i_{G}, i_{H}\right)$ and then, using this value,

$$
\begin{aligned}
& \hat{\hat{i}}_{D, A}=\hat{f}_{D}\left(\hat{i}_{A}, i_{E}, i_{G}, i_{H}\right) \\
& \hat{\hat{i}}_{E, A}=\hat{f}_{E}\left(\hat{i}_{A}, i_{D}, i_{G}, i_{H}\right) \\
& \hat{\hat{i}}_{G, A}=\hat{f}_{G}\left(\hat{i}_{A}, i_{D}, i_{E}, i_{H}\right) \\
& \hat{\hat{i}}_{H, A}=\hat{f}_{H}\left(\hat{i}_{A}, i_{D}, i_{E}, i_{G}\right)
\end{aligned}
$$

where e.g. $\hat{\hat{i}}_{D, A}$ means the prediction for sensor D obtained by replacing $i_{A}$ with $\hat{i}_{A}$ as inputs of $\hat{f}_{D}$.

The errors $\hat{i}-i$ do not change significantly with respect to $\hat{i}-i$ until we substitute $\mathrm{H}$ with its model. Think for example at the prediction of the output of the sensor D: the prediction for the current flowing in D is wrong both if only the input $i_{H}$ (case $\hat{i}_{D}$ ) and if the inputs $i_{H}$ and $\hat{i}_{A}$ are wrong (case $\hat{\hat{i}}_{D}$ ). 
But when we substitute $\mathrm{H}$ with its model the high estimation errors for the good working sensors A, D, E and G disappear (Fig. 6). This is because e.g. $\hat{\hat{i}}_{D, H}$ now correctly estimates $i_{D}$ because the input arguments of $\hat{i}_{D, H}$ are all correct, including $\hat{i}_{H}$.

Having determined the malfunctioning sensor, the next step is to compensate for the error until the sensor is substituted. In fact, the neural network which had been previously trained for the prediction of the gas components' concentration needs five inputs and it surely will give wrong predictions if one input is incorrect. In our approach we simply use the estimate $\hat{i}$ given by the other sensors to substitute the damaged one. Because of the sensors' correlation, in this way the concentration predictions are only slightly affected.

\section{Conclusions}

The proposed approach to reliability control exploits the strong correlation of the sensors' array conductances. It uses five MLP networks, each of them estimating the current flowing in one sensor as a non-linear function of the currents flowing in the other ones. In case a fault has been detected, the estimation replaces the response of the damaged sensor so that the subsequent phase of concentration prediction does not have to be interrupted.

On the basis of this neural cross-monitoring of the sensors' working state, various criteria can be implemented in order to detect different kind of faults. The reported experimental results are related to a transient fault detection by considering the actual estimated error levels as a signature of the correct working state of sensors. As a further application, long time drifts can be detected by checking whether the estimation errors are Normal distributed.

\section{Acknowledgments}

This work was partially supported by the Italian National Research Council (CNR) in the frame of the project MADESS II. 


\section{References}

[1] S. Marco, A. Ortega, A. Pardo, and J. Samitier. Gas identification with tin oxide sensor array and self organizing maps: adaptive correction of sensor drifts. IEEE Trans. on Instrum. and Meas., 47:316-321, 1998.

[2] Y. Kato, K. Yoshokawa, and M. Kitora. Temperature-dependent dynamic response enables the qualification and quantification of gases by a single sensor. Sensors and Actuators B, 40:33-37, 1997.

[3] S.W. Moore, J.W. Gardner, E.L. Hines, U. Weimar, and W. Gopel. A modified multilayer perceptron model for gas mixture analysis. Sensors and Actuators $B$, 15-16:344-348, 1993.

[4] E. Llobet, J. Brezmes, X. Vilanova, J. Sueiras, and X. Correig. Qualitative and quantitative analysis of volatile organic compounds using transient and steady state responses of a thick film tin oxide gas sensor array. Sensors and Actuators $B, 41: 13-21,1997$.

[5] H. Sungreen, F. Winquist, I. Lukkari, and I. Lundstrom. Artificial neural networks and gas sensors arrays: quantification of individual components in a gas mixture. Meas. Sci. Technol., 2:464-469, 1992.

[6] U. Hoefer, H. Bottner, A. Felske, G. Kuhner, K. Steiner, and G. Sulz. Thin-film $\mathrm{SnO}_{2}$ sensor arrays controlled by variation of contact potential-asuitable tool for chemometric gas mixture analysis in the tlv range. Sensors and Actuators $B, 44: 429-433,1997$.

[7] G. Kraus, A. Hierlemann, G. Gauglitz, and W. Gopel. Analysis of complex gas mixtures by pattern recognition with polymer based quartz microbalance sensor array. In Transducer 95 and Eurosensors IX, pages 675-678, Stockholm, June 1995.

[8] M. Schweizer-Berberich, A. Hierlemann, K. Bodenhofer, J. Mitrovics, T. Kerner, U. Weimar, and W. Gopel. Evaluation of dynamic sensor signals by artificial neural networks. In Transducer 95 and Eurosensors IX, pages 679-682, Stockholm, June 1995.

[9] M. Corte, F. Masulli, M. Riani, M. Pardo, G. Faglia, and G. Sberveglieri. Estimation of gas concentrations in a mixture using a neural dynamical model. In Proc. of the 7th International Meeting on Chemical Sensors, pages 770-772, Beijing, China, July 1998.

[10] M. Pardo, G. Faglia, G. Sberveglieri, M. Corte, F. Masulli, and M. Riani. A time delay neural network for estimation of gas concentrations in a mixture. Sensors and Actuators $B$ (to be published).

[11] M. Johnston, K. Kimura, R. Hinkle, and S. Melville. Virtual analyzers help refiners meet rfg specifications. Hydrocarbon Processing, pages 57-61, 1999. 
[12] G. Sberveglieri. Recent developments in semiconducting film gas sensors. Sensors and Actuators B, 23:103-109, 1995.

[13] G. Sberveglieri, L. Depero, S. Groppelli, and P. Nelli. $W O_{3}$ sputtered thin films for $N O_{X}$ monitoring. Sensors and Actuators B, 26-27:89-92, 1995.

[14] G. Cybenko. Approximation by superpositions of a sigmoidal function. Mathematics of Control Signals and Systems, 2:303-314, 1989. 


\section{Figure captions}

Figure 1: Sensor A: Temporal plot of measured and estimated currents for the test set.

Figure 2: Sensor H: Scatter of measured and estimated currents for the test set.

Figure 3: Scheme of the MLP-based reliability control system.

Figure 4: The simulated malfunctioning of sensor $\mathrm{H}$ (points 19, 20, 21).

Figure 5: Differences in nA between the measured and estimated current. From top left clockwise $\left|i_{A}-\hat{i}_{A}\right|,\left|i_{D}-\hat{i}_{D}\right|,\left|i_{E}-\hat{i}_{E}\right|,\left|i_{H}-\hat{i}_{H}\right|,\left|i_{G}-\hat{i}_{G}\right|$.

Figure 6: Differences in $\mathrm{nA}$ between the measured and estimated current substituting $\mathrm{H}$ with its model. From top left clockwise $\left|i_{A}-\hat{\hat{i}}_{A, H}\right|,\left|i_{D}-\hat{\hat{i}}_{D, H}\right|$, $\left|i_{E}-\hat{\hat{i}}_{E, H}\right|,\left|i_{H}-\hat{\hat{i}}_{G, H}\right|$. 


\section{Table captions}

Table 1: Mean square errors of neural estimators $\left(\mu A^{2}\right)$. 


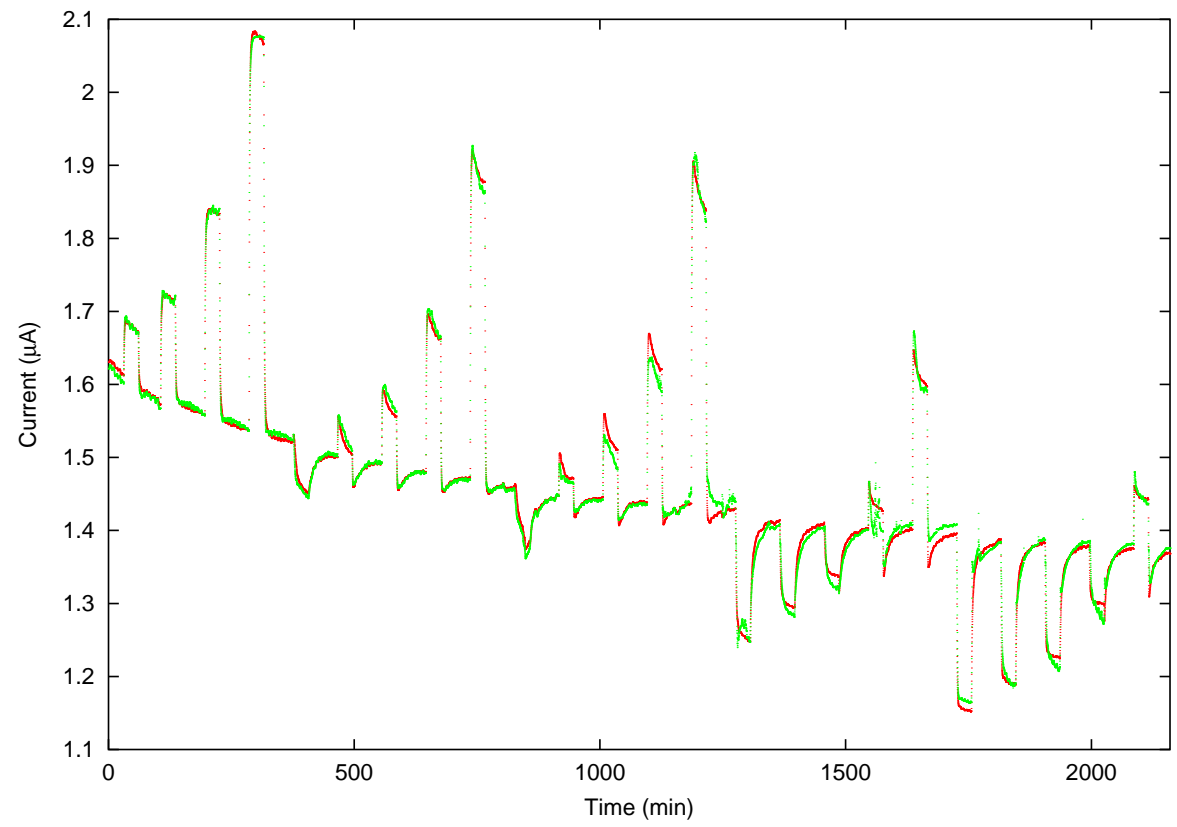

Figure 1. 


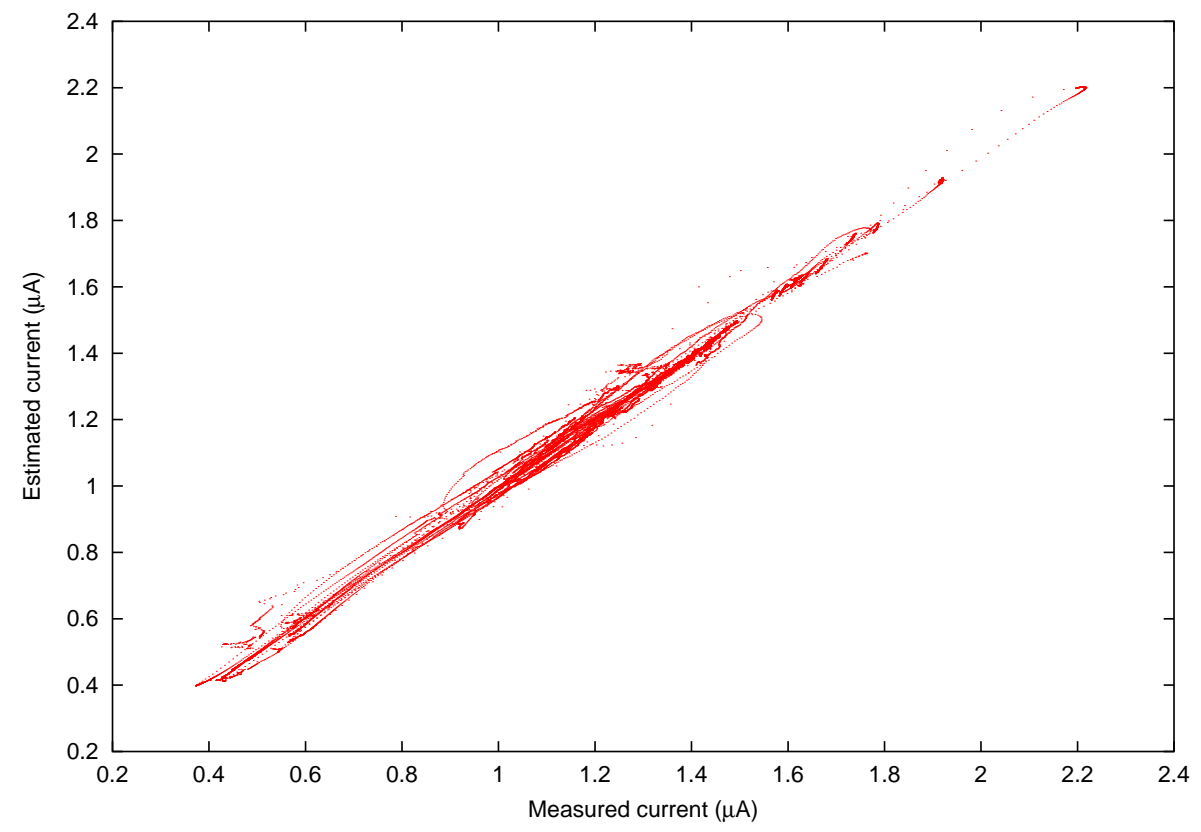

Figure 2. 


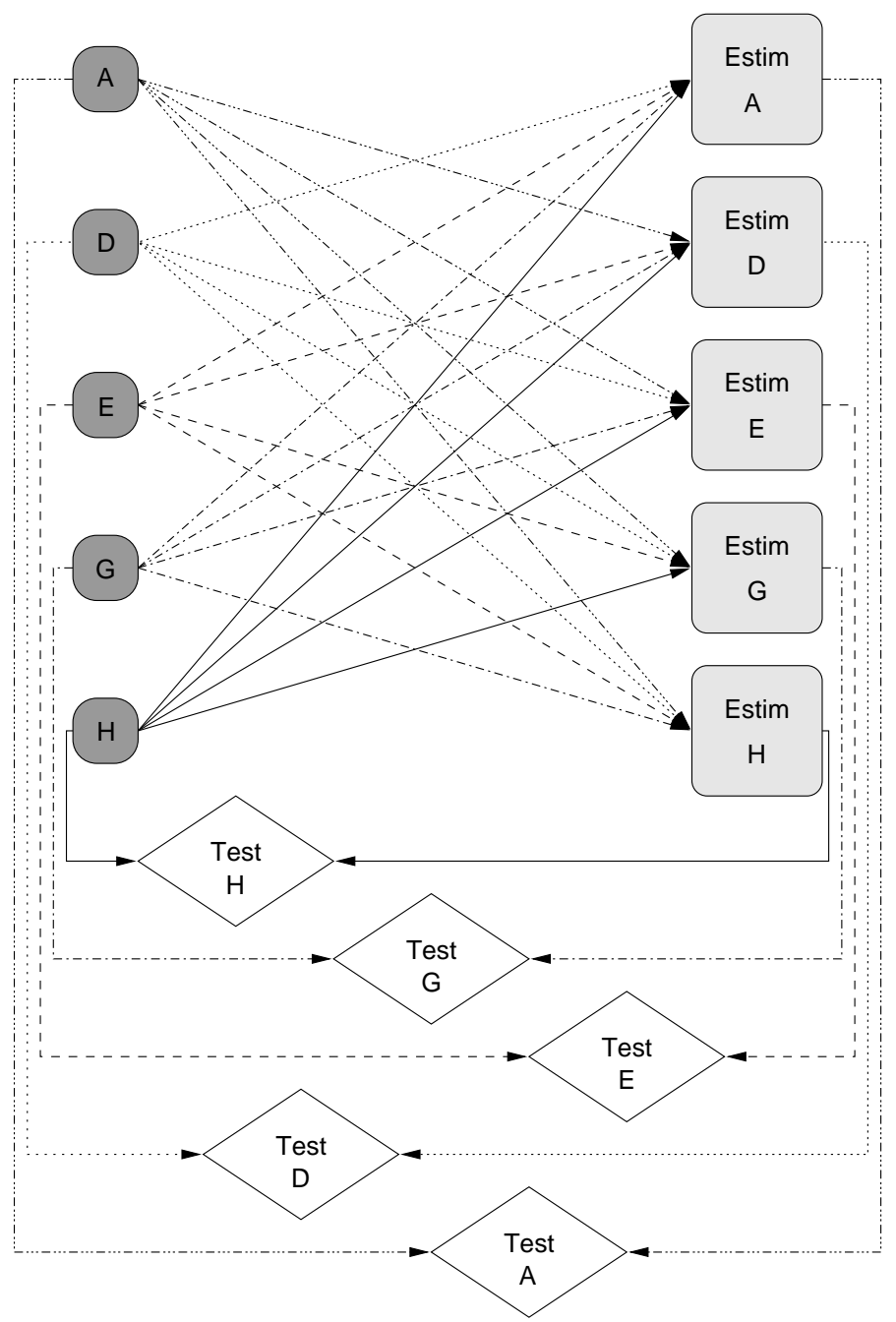

Figure 3. 


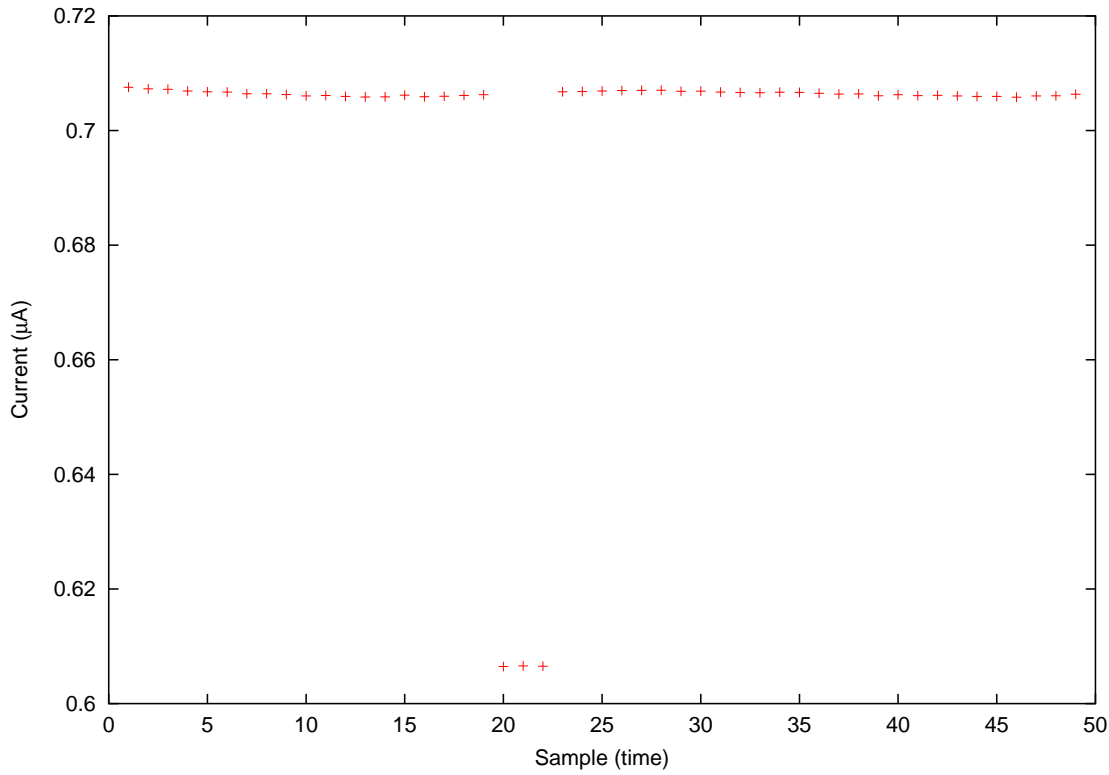

Figure 4. 

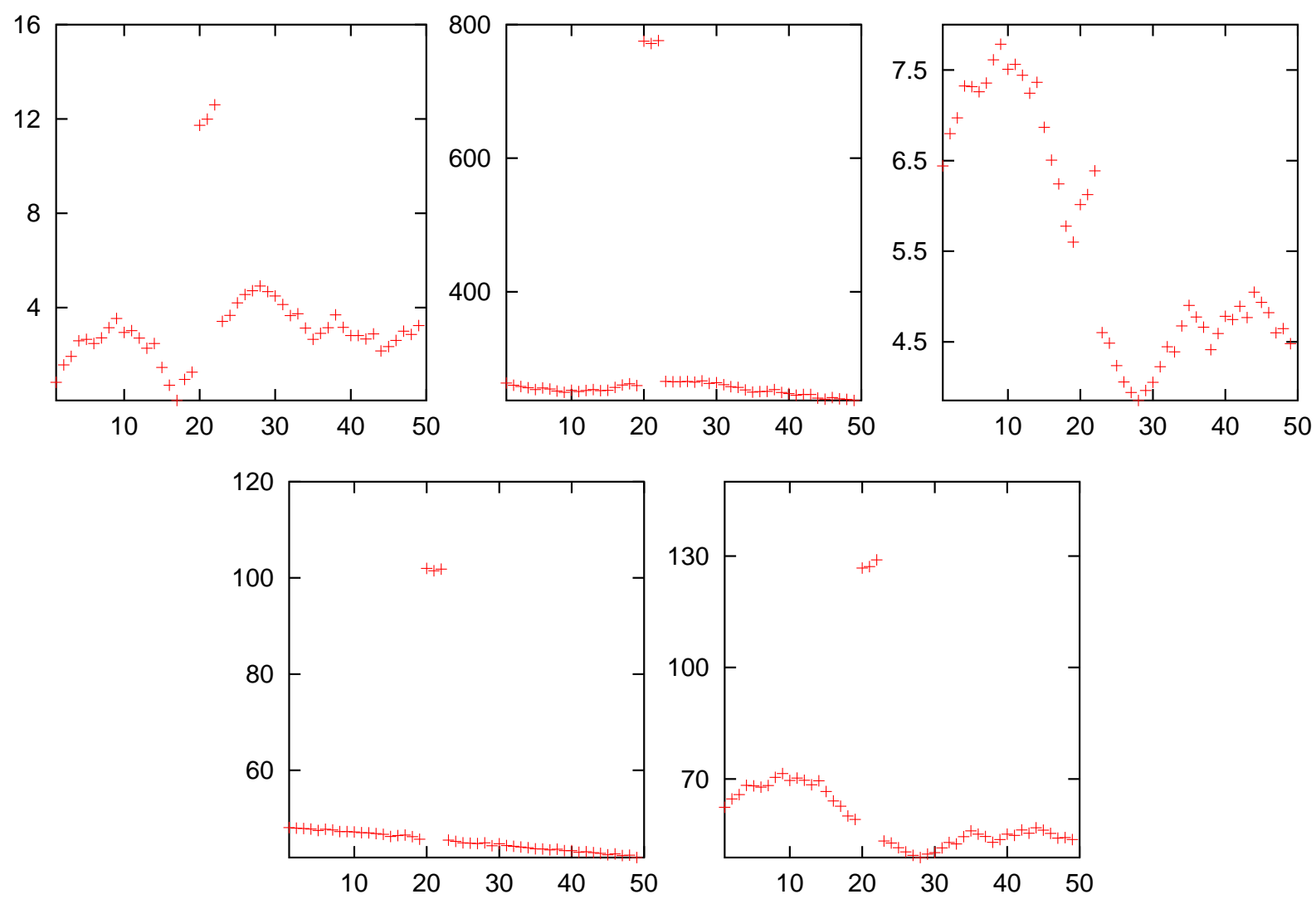

Figure 5. 

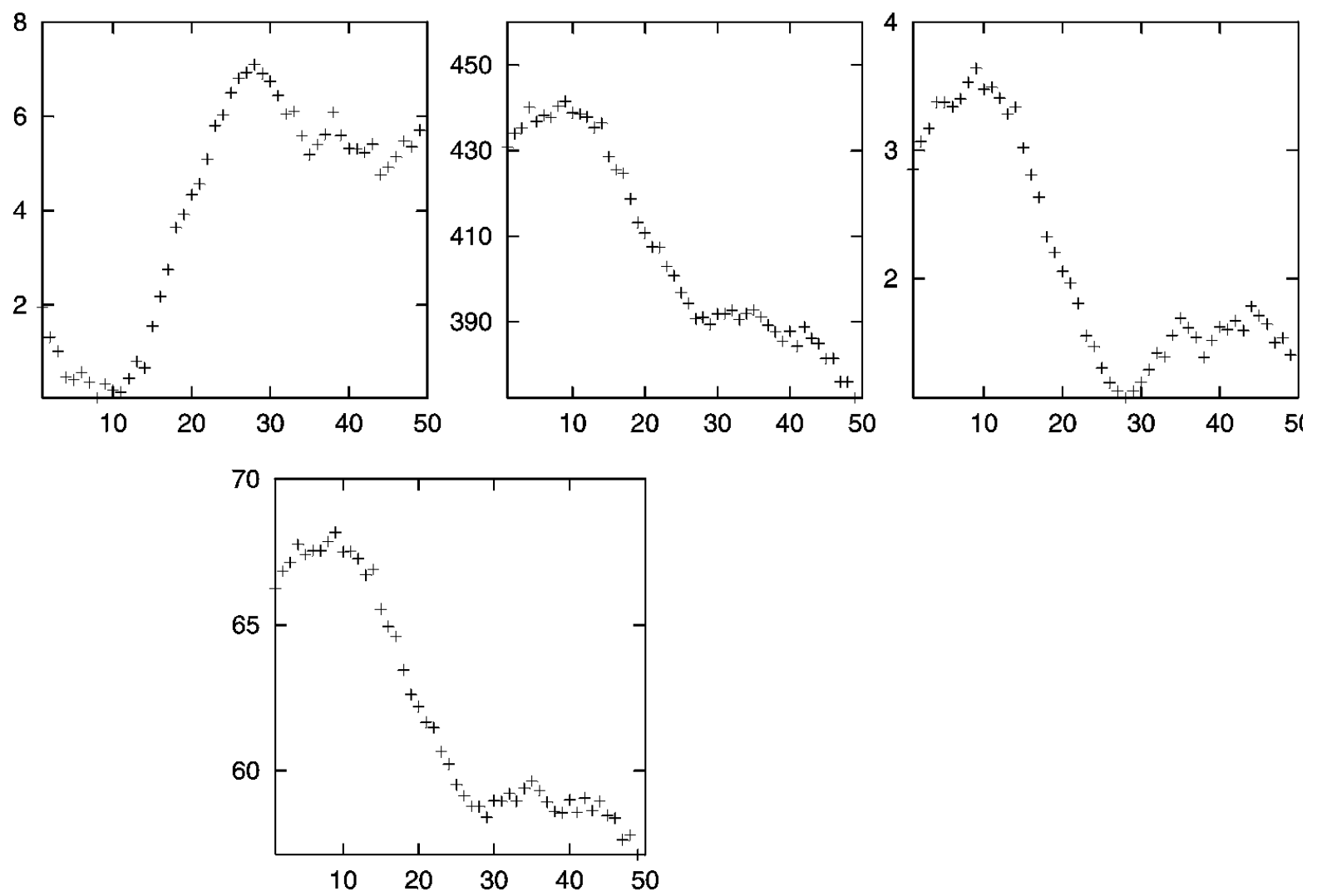

Figure 6 . 
Table 1

\begin{tabular}{|c|c|c|}
\hline sensor & training set & test set \\
\hline \hline $\mathrm{A}$ & $8 \cdot 10^{-5}$ & $1 \cdot 10^{-4}$ \\
\hline $\mathrm{D}$ & $4 \cdot 10^{-3}$ & $6 \cdot 10^{-3}$ \\
\hline $\mathrm{E}$ & $1 \cdot 10^{-5}$ & $4 \cdot 10^{-5}$ \\
\hline $\mathrm{G}$ & $6 \cdot 10^{-4}$ & $4 \cdot 10^{-4}$ \\
\hline $\mathrm{H}$ & $2 \cdot 10^{-4}$ & $6 \cdot 10^{-4}$ \\
\hline
\end{tabular}

